\title{
Outcome of pulmonary endarterectomy in symptomatic chronic thromboembolic disease
}

\author{
Dolores Taboada', Joanna Pepke-Zaba', David P. Jenkins², Marius Berman², \\ Carmen M. Treacy', John E. Cannon ${ }^{1}$, Mark Toshner ${ }^{1}$, John J. Dunning ${ }^{2}$, \\ Choo $\mathrm{Ng}^{2}$, Steven S. Tsuil ${ }^{2}$ and Karen K. Sheares ${ }^{1}$
}

\author{
Affiliations: \\ ${ }_{1}^{1}$ Pulmonary Vascular Diseases Unit, Papworth Hospital, Cambridge, UK. \\ ${ }^{2}$ Dept of Cardiothoracic Surgery, Papworth Hospital, Cambridge, UK.
}

\section{Correspondence:}

Karen K. Sheares, Pulmonary Vascular Diseases Unit, Papworth Hospital, Papworth Everard, Cambridge CB23 3RE, UK. E-mail: Karen.Sheares@apapworth.nhs.uk

ABSTRACT Chronic thromboembolic disease is characterised by persistent pulmonary thromboembolic occlusions without pulmonary hypertension. Early surgical treatment with pulmonary endarterectomy may improve symptoms and prevent disease progression. We sought to assess the outcome of pulmonary endarterectomy in symptomatic patients with chronic thromboembolic disease.

Patients with symptomatic chronic thromboembolic disease and a mean pulmonary artery pressure $<25 \mathrm{mmHg}$ at baseline with right heart catheterisation and treated with pulmonary endarterectomy between January 2000 and July 2013 were identified. Patients were reassessed at 6 months and at 1 year following surgery.

A total of 42 patients underwent surgery and the median length of stay in hospital was 11 days. There was no in-hospital mortality but complications occurred in $40 \%$ of patients. At 1 year, following surgery, $95 \%$ of the patients remained alive. There was a significant symptomatic improvement with $95 \%$ of patients in the New York Heart Association functional classes I or II at 6 months. There was a significant improvement in quality of life assessed by the Cambridge pulmonary hypertension outcome review questionnaire.

In this carefully selected cohort of chronic thromboembolic disease patients, pulmonary endarterectomy resulted in significant improvement in symptoms and quality of life. Appropriate patient selection is paramount given the known surgical morbidity and mortality, and surgery should only be performed in expert centres.

@ERSpublications

Pulmonary endarterectomy improves symptoms and quality of life in patients with chronic thromboembolic disease http://ow.ly/AeECt

For editorial comments see page 1401.

Received: March 152014 | Accepted after revision: July 292014 | First published online: Sept 182014

Support statement: This research was supported by the National Institute for Health Research (NIHR) Cambridge Biomedical Research Centre.

Conflict of interest: Disclosures can be found alongside the online version of this article at erj.ersjournals.com 


\section{Introduction}

Chronic thromboembolic pulmonary hypertension (CTEPH) is a serious condition that results from insufficient resolution of thromboemboli in the pulmonary arteries. It is characterised by vascular remodelling causing persistent elevation of pulmonary arterial pressure (PAP) and pulmonary vascular resistance (PVR) leading to right ventricular failure [1]. This is a progressive disease, usually fatal when left untreated and mortality correlates with mean PAP at diagnosis. Historical data, preceding the current treatment era, showed a poor survival of around $10 \%$ at 5 years in patients with a mean PAP $>50 \mathrm{mmHg}$ and a fairly good prognosis in those with a mean PAP $<30 \mathrm{mmHg}[2,3]$.

The treatment of choice, and only potentially curative option, is surgical disobliteration of the pulmonary arteries by pulmonary endarterectomy (PEA) [3]. Appropriate decision making to determine operability on CTEPH patients can be challenging and takes into account the patient's symptoms and functional impairment, severity of pulmonary hypertension $(\mathrm{PH})$, as well as surgical accessibility of thromboembolic lesions and coexistent comorbidities [4, 5].

PEA was first performed at Papworth Hospital (Cambridge, UK) in 1997 and the hospital was commissioned to provide a PEA service for the UK in 2000. Since then, more than a thousand operations have been performed with increased institutional and surgical experience leading to improved outcome in line with $4.7 \%$ in-hospital mortality reported by the first international CTEPH prospective registry [6]. Inhospital mortality at our centre is now 2-3\% [7] and long-term survival has also improved with $90 \%$ conditional survival at 5 years post-surgery [8].

Improved survival and reduced associated morbidity in high volume experienced PEA centres worldwide has led to successful PEA in more challenging patient groups, such as elderly patients [9] and patients with more distal segmental disease [10]. Similarly, at the other end of the risk spectrum, PEA surgery is also being considered in patients with significant chronic vascular occlusions but near normal pulmonary haemodynamics at rest; we have defined this group as "chronic thromboembolic disease" (CTED). The rationale is to improve symptoms and exercise tolerance and hopefully, with early intervention, prevent the development of secondary small-vessel vasculopathy and right heart failure [10-13]. We have been encouraged to extend the indication for PEA to patients with CTED as the results of surgery have improved, and in particular our previous finding of no decline in cognitive function in the PEACOG study [14]. However, the outcome of PEA in CTED patients has not been formally reviewed previously.

The aim of this study is to assess the outcome of PEA in symptomatic patients with extensive CTED in the absence of $\mathrm{PH}$ as currently defined (mean PAP $>25 \mathrm{mmHg}$ ).

\section{Patients and methods}

\section{PEA pathway}

All new CTEPH patients referred to Papworth Hospital for PEA are discussed at our weekly multidisciplinary meeting with pulmonary hypertension physicians, radiologists and PEA surgeons. Patients are selected for surgery based in the presence of symptoms and exercise limitation, which cannot be explained by left heart disease according to results of an echocardiogram or by lung parenchymal disease according to lung function tests and imaging. Surgical accessibility of thromboembolic lesions is assessed using at least two imaging modalities (conventional pulmonary angiogram, computed tomography pulmonary angiogram (CTPA), magnetic resonance pulmonary angiography). Data are entered prospectively in a dedicated $\mathrm{PH}$ and surgical database.

Patients in whom PEA is considered are invited to Papworth Hospital for pre-operative discussion with a PEA nurse specialist and the lead PEA surgeon. For patients with CTED, the options of surgery for symptomatic benefit or the alternative of conservative treatment with regular follow-up are discussed. The minimum risk quoted for the possibility of a serious complication or death is $5 \%$. Patients who decide to undergo PEA surgery have a filter fitted in the inferior vena cava prior to operation. PEA is performed following similar principles to those used by the University of California San Diego Medical (San Diego, CA, USA) [15].

Patients were invited to come to Papworth Hospital 3-6 months after PEA for a comprehensive review that includes New York Heart Association (NYHA) functional class, 6-min walk test (6MWT) and a right heart catheterisation (RHC). Health related quality of life (QoL) is measured using the Cambridge pulmonary hypertension outcome review (CAMPHOR) questionnaire. This is a disease-specific questionnaire comprising three sections evaluating symptoms, activity levels and QoL; it is negatively weighted with the higher scores indicating worse QoL and greater functional limitation [16]. The same variables excluding a RHC are assessed again at 12 months after PEA. Follow-up imaging was also performed at 3-6 months, but is not reported here. 
CTED cohort

We have identified, from our database, patients with CTED and a mean PAP $<25 \mathrm{mmHg}$ at baseline RHC that underwent PEA at Papworth Hospital between January 1, 2000 and July 1, 2013. Baseline characteristics, perioperative data and post-operative assessment results were collected from both database and patient's notes. Date of last venous thromboembolism (VTE) was collected from patient's notes. Time from last VTE to diagnosis was defined as time from last pulmonary embolism to diagnosis of CTED. The date of the CTED diagnosis date was defined by the date of baseline RHC. In-hospital survival was defined as survival to discharge from hospital. The length of in-hospital and intensive care unit (ICU) stay was calculated from the date of surgery to discharge. Major complications were defined as having at least one of following: in-hospital death, return to the operating theatre for any reason, re-intubation, tracheostomy, pneumothorax, intracranial bleeding and any minor complication resulting in prolonged hospital stay over 2 weeks. Database lock was performed on January 31, 2014.

\section{Statistical analysis}

Statistical analysis was performed using STATA version 12 (StataCorp LP, College Station, TX, USA). Categorical variables were expressed as a proportion. Continuous variables were tested for adherence to a normal distribution by Shapiro-Wilk test for normal data and expressed as mean \pm SD or median (interquartile range (IQR)).

Categorical data were compared using Fisher's exact test. Paired student's t-test was used to compare means from normally distributed variables. Wilcoxon signed-rank tests were used to compare data that cannot be approximated by normal distribution. Values of $\mathrm{p}<0.05$ were considered significant. This service evaluation study was approved by the Research and Development Department at Papworth Hospital (reference number S01864).

\section{Results}

Between January 2000 and July 2013, 1019 patients underwent PEA at Papworth Hospital. Of those, 42 patients fulfilled the criteria of having CTED.

\section{Demographics}

All patients had evidence of chronic thromboembolic obstruction on CTPA despite at least 6 months of effective anticoagulation with documented treatment compliance. None of the patients were on any pulmonary vasodilator therapy at the time of haemodynamic assessment. Baseline characteristics are displayed in tables 1 and 2.

Mean age of the patients was $49 \pm 16$ years and $60 \%$ of the patients were female. $90 \%$ of the patients had a documented history of VTE, with a third of patients having more than one episode. All patients were symptomatic, the most frequent symptom was exertional breathlessness and all presented with NYHA functional class II or III limitation. Excluding those patients having combined surgical procedures, no evidence of significant cardiopulmonary comorbidities was found to explain the patient's symptoms (table 2).

At RHC the mean PAP and PVR were mildly elevated at median (IQR) 21 (5) mmHg and 164 (104) dyn $\cdot \mathrm{s} \cdot \mathrm{cm}^{-5}$, respectively, not fulfilling criteria for current definition of pulmonary hypertension and cardiac index was at the lower limit of normal at $2.6 \pm 0.5 \mathrm{~L} \cdot \mathrm{min}^{-1} \cdot \mathrm{m}^{-2}$. Patients scored high in all three domains of the CAMPHOR questionnaire indicating a poor-health related QoL.

Comorbidities and risk factors for VTE are summarised table 3. 22 (52\%) patients had at least one comorbidity that could also contribute to breathlessness and reduced exercise capacity.

All patients were offered inferior vena cava filter insertion prior to surgery and it was successfully fitted in 36 patients.

\section{Surgical outcomes}

All patients had significant burden of bilateral thromboembolic disease. The endarterectomy specimens as per Jamieson classification are summarised in table 4, with the majority of patients having bilateral type 2 disease and samples of the PEA specimens from the patients are given in figure 1.

Concomitant surgical procedures were performed in four patients (coronary fistula closure $n=1$, tricuspid valve repair for severe tricuspid regurgitation due to previous endocarditis $n=2$, coronary artery bypass grafting $\mathrm{n}=1$ ). One patient had a previous sternotomy for epicardial pacing.

All patients were alive at discharge after a median (IQR) in-hospital stay of 11 (6) days and ICU stay of 3 (2) days. A list of all complications including major and minor ones is shown in figure 2. The most common was supraventricular arrhythmias with six patients requiring cardioversion. Most wound problems 


\section{TABLE 1 Clinical characteristics of patient at diagnosis}

\begin{tabular}{|c|c|}
\hline Patients & 42 \\
\hline Age years & $49 \pm 16$ \\
\hline Female \% & 60 \\
\hline Body mass index & $30 \pm 7$ \\
\hline Previous VTE \% & $\overline{90}$ \\
\hline Time from VTE to CTED diagnosis months & $14(27)$ \\
\hline Time from CTED diagnosis to PEA ${ }^{\#}$ months & $8(5)$ \\
\hline Time from PEA to first visit months & $6 \pm 3$ \\
\hline Time from PEA to second visit ${ }^{+}$months & $13 \pm 2$ \\
\hline NYHA functional class II & 20 \\
\hline NYHA functional class III & 22 \\
\hline Creatinine $\mu \mathrm{mol} \cdot \mathrm{L}^{-1}$ & $90 \pm 49$ \\
\hline $\mathrm{N}$-terminal pro-brain natriuretic peptide ${ }^{\S} \mathrm{pg} \cdot \mathrm{mL}^{-1}$ & 51.5 \\
\hline \multicolumn{2}{|l|}{ Right heart catheterisation } \\
\hline Patients & 42 \\
\hline PAP mean $\mathrm{mmHg}$ & $21(5)$ \\
\hline $\mathrm{SVO}_{2} \%$ & $72 \pm 6$ \\
\hline Cardiac index $\mathrm{L} \cdot \mathrm{min}^{-1} \cdot \mathrm{m}^{-2}$ & $2.6 \pm 0.5$ \\
\hline Ppaw $\mathrm{mmHg}$ & $9 \pm 3$ \\
\hline PVR dyn $\cdot s \cdot \mathrm{cm}^{-5}$ & $164(104)$ \\
\hline \multicolumn{2}{|l|}{ 6-min walk test } \\
\hline Patients ${ }^{f}$ & 37 \\
\hline Distance $\mathrm{m}$ & $372+117$ \\
\hline Baseline $\mathrm{SpO}_{2} \%$ & $97 \pm 1.8$ \\
\hline Minimal exercise $\mathrm{SpO}_{2} \%$ & $91(6)$ \\
\hline \multicolumn{2}{|l|}{ CAMPHOR $\# \#$} \\
\hline Patients & 36 \\
\hline Symptoms & $15(13)$ \\
\hline Activity & $10(9)$ \\
\hline Quality of life & $14(14)$ \\
\hline
\end{tabular}

Data are presented as $n$, mean \pm SD or median (interquartile range), unless otherwise stated. VTE: venous thromboembolism; CTED: chronic thromboembolic disease; PEA: pulmonary endarterectomy; NYHA: New York Heart Association; PAP: pulmonary artery pressure; $\mathrm{SvO}_{2}$ : mixed venous oxygen saturation; Ppaw: pulmonary artery wedge pressure; PVR: pulmonary vascular resistance; $\mathrm{SpO}_{2}$ : arterial oxygen saturation measured by pulse oximetry; CAMPHOR: Cambridge pulmonary hypertension outcome review. \#: CTED diagnosis defined by the date of baseline right heart catheterisation; ${ }^{\circledR}: n=39 ;{ }^{+}: n=31 ;{ }^{\S}: n=20 ;{ }^{f}$ : five patients had shuttle walk instead of 6-min walk test and data was not included; ${ }^{\# \#}$ : CAMPHOR questionnaire was implemented in 2004.

were minor and related to superficial infection, with the exception of two patients that required vacuum assisted closure. Six patients required post-operative noninvasive ventilatory support with continuous positive airway pressure, mainly as treatment for hypoxia due to reperfusion injury. Major complications were observed in $17(40 \%)$ patients. Three patients had to return to theatre (for cardiac tamponade, haemothorax and supraventricular tachycardia with no cardiac output). Four patients had a pneumothorax with two requiring chest drain insertion. There were six small subdural haematomas not requiring intervention. Three patients required re-intubation (reperfusion injury $n=2$ and resternotomy $n=1$ ). The first patient of the cohort had difficulty weaning and required a tracheostomy.

By January 31, 2014, two (5\%) patients had died after discharge from hospital. One was a female aged 77 years who died at her local hospital 29 days after PEA and the post mortem confirmed acute pulmonary embolism. No inferior vena cava filter was inserted prior to surgery, due to unfavourable venous anatomy, and anticoagulation was temporarily interrupted due to a small subdural haematoma. The second patient was a male aged 47 years with a psychiatric illness who was diagnosed with a small recurrent pulmonary embolism 4 months after surgery. He died suddenly at home 115 days after PEA. A post mortem examination was not performed and his death was listed as an unexplained sudden death.

\section{Post-operative assessment}

At the time of the database lock, at the end of January 2014, 39 patients had a first comprehensive review at, on average, 6 months post-surgery and 31 patients were again reviewed 1-year post-PEA. Post-operative results are displayed in table 5 . 


\section{TABLE 2 Echocardiography and lung function tests}

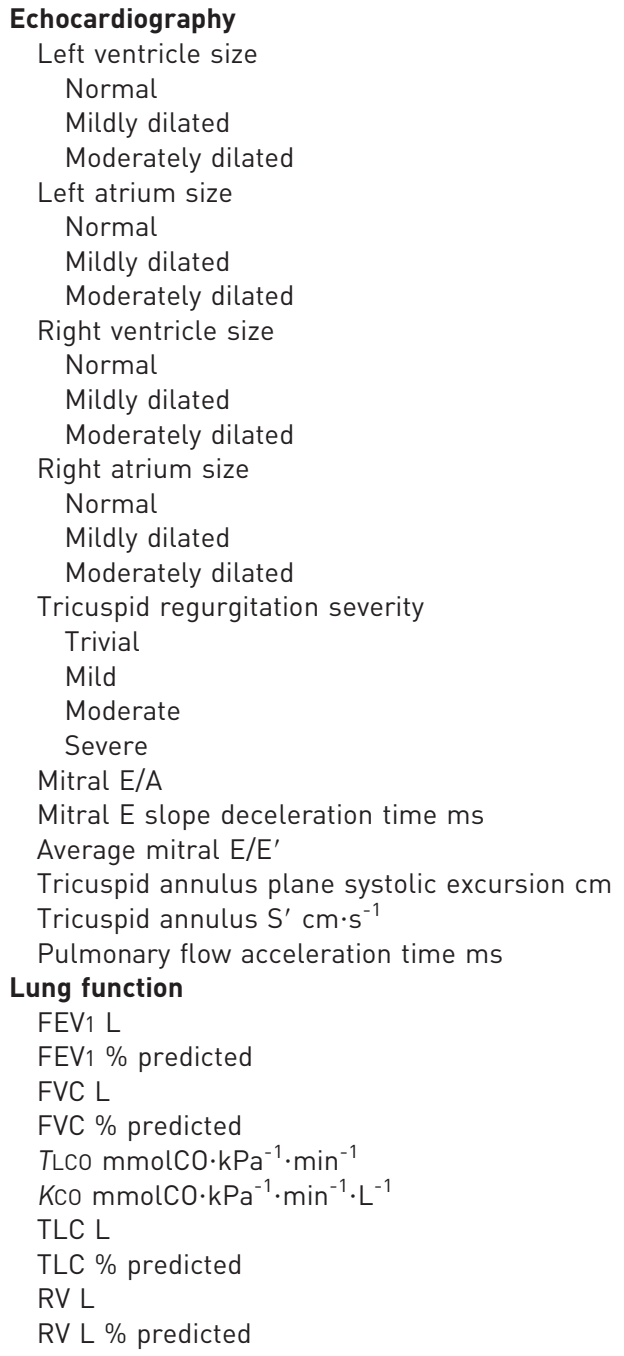

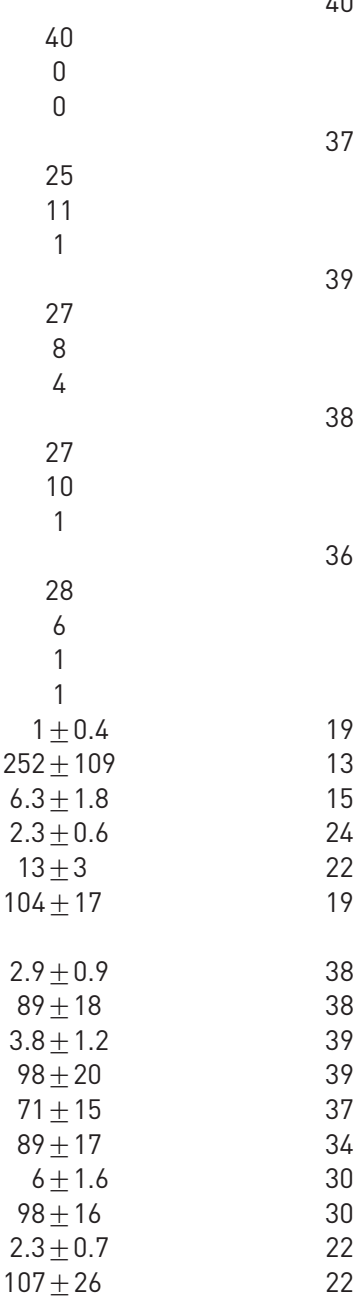

Data are presented as $\mathrm{n}$ or mean \pm SD. FEV1: forced expiratory volume in $1 \mathrm{~s}$; FVC: forced vital capacity; TLCO: transfer factor of the lung for carbon monoxide; KCO: transfer coefficient of the lung for carbon monoxide; TLC: total lung capacity; RV: residual volume.

TABLE 3 Comorbidities and risk factors for venous thromboembolism

Body mass index $>30$

Thrombophilia"

Asthma/chronic obstructive pulmonary disease

Inflammatory bowel disease

Previous cancer

Other exercise limitation

Tricuspid valve endocarditis

Previous pacemaker

7
7
3
3
3
3
1

Data are presented as $n$. " : antiphospholipid syndrome $(n=2)$, lupus anticoagulant $(n=1)$, protein $C$ deficiency $(n=2)$, factor $V$ Leiden $(n=2)$; ${ }^{\circ}$; polio sequel $(n=1)$, peripheral artery disease $(n=1)$, previous spine surgery $(n=1)$. 


\section{TABLE 4 Jamieson surgical classification of pulmonary endarterectomy specimens}

\section{Same types in both lungs}

Bilateral type 2

Bilateral type 3

Different types between lungs"

Type 1

Type 2

No data available

\#: disease type was different on each side, the patient was categorised with the highest type as per MADANI et al [10].

There was a significant improvement in symptoms with $95 \%$ of patients returning to NYHA functional class I and II during the first year post-surgery (figs $3 a$ and 4a). A significant improvement in the total score (median (IQR)) and all three domains of the CAMPHOR questionnaire was observed at 6 months and sustained at 1 year (figs $3 \mathrm{~b}$ and $4 \mathrm{c}$ ); the total score went from 40 (33) at baseline to 11 (30) at 6-months post-PEA $(\mathrm{p}<0.001)$ and $11(37)$ at 1 -year post PEA $(\mathrm{p}<0.001)$; symptoms went from 15 (13) to 4 (12) $(\mathrm{p}<0.001)$ and $5(12)(\mathrm{p}<0.001)$, respectively; activity went from $10(9)$ to $5(6)(\mathrm{p}=0.002)$ and $4(11)$ $(\mathrm{p}<0.001)$, respectively; and QoL went from $14(14)$ to $2(11)(\mathrm{p}=0.003)$ and $1(12)(\mathrm{p}=0.001)$, respectively. There was a reduction in the mean PAP from 21 (5) $\mathrm{mmHg}$ at baseline to 18 (5) $\mathrm{mmHg}$ at 6-months post-PEA $(\mathrm{p}=0.005)$ and PVR went from $164(104) \mathrm{dyn} \cdot \mathrm{s} \cdot \mathrm{cm}^{-5}$ to $128(60) \mathrm{dyn} \cdot \mathrm{s} \cdot \mathrm{cm}^{-5}$ $(\mathrm{p}<0.001)$, respectively. However, no change in cardiac index (mean $\pm \mathrm{SD}$ ) was observed, which was $2.6 \pm 0.5 \mathrm{~L} \cdot \mathrm{min}^{-1} \cdot \mathrm{m}^{-2}$ at baseline versus $2.5 \pm 0.4 \mathrm{~L} \cdot \mathrm{min}^{-1} \cdot \mathrm{m}^{-2}$ at 6 -months post-PEA (nonsignificant). The 6 -min walking distance $(6 \mathrm{MWD})$ (mean $\pm \mathrm{SD}$ ) improved significantly at 6 months and this improvement was maintained at 1 year $(372 \pm 117 \mathrm{~m}$ at baseline versus $413 \pm 90 \mathrm{~m}$ at 6 -months post-PEA $(\mathrm{p}=0.04)$ and $421 \pm 113 \mathrm{~m}$ at 1 -year post-PEA $(\mathrm{p}=0.008)$ ). Individual changes in $6 \mathrm{MWD}$ are illustrated in figure $4 \mathrm{~b}$. Minimal oxygen saturation on exercise improved compared to baseline and this was significant at 1-year

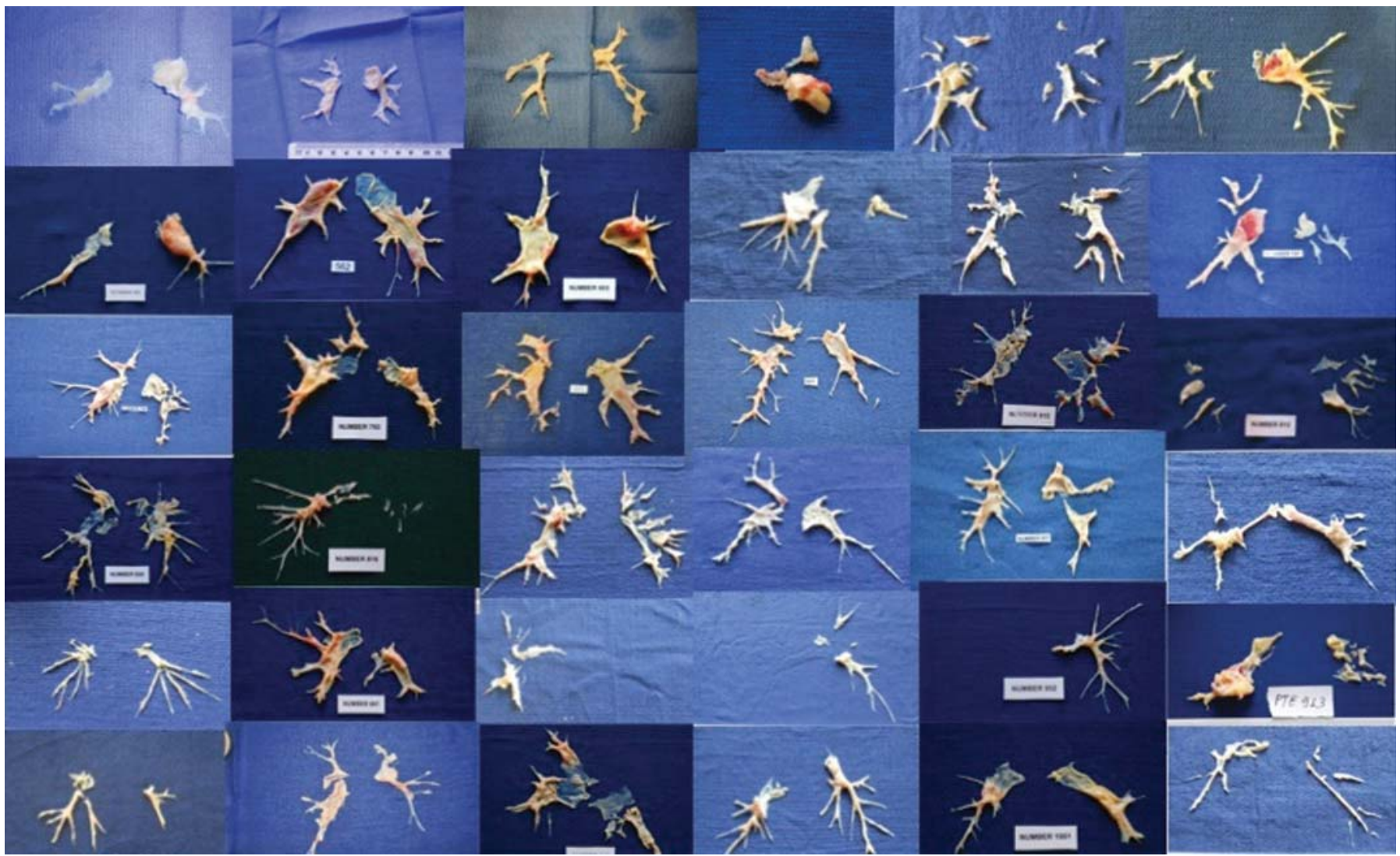

FIGURE 1 Samples of the pulmonary endarterectomy specimens taken from the patients included in the study, 36 pictures were available, six pictures were unavailable. 
FIGURE 2 Perioperative complications of pulmonary endarterectomy encountered by the patients in the study. SVT: supraventricular tachycardia; CPAP: continuous positive airway pressure; SDH: subdural haematoma; VTE: venous thromboembolism; LRTI: lower respiratory tract infection. ": severe vertigo, lumbar plexus lesion, diabetic ketoacidosis.

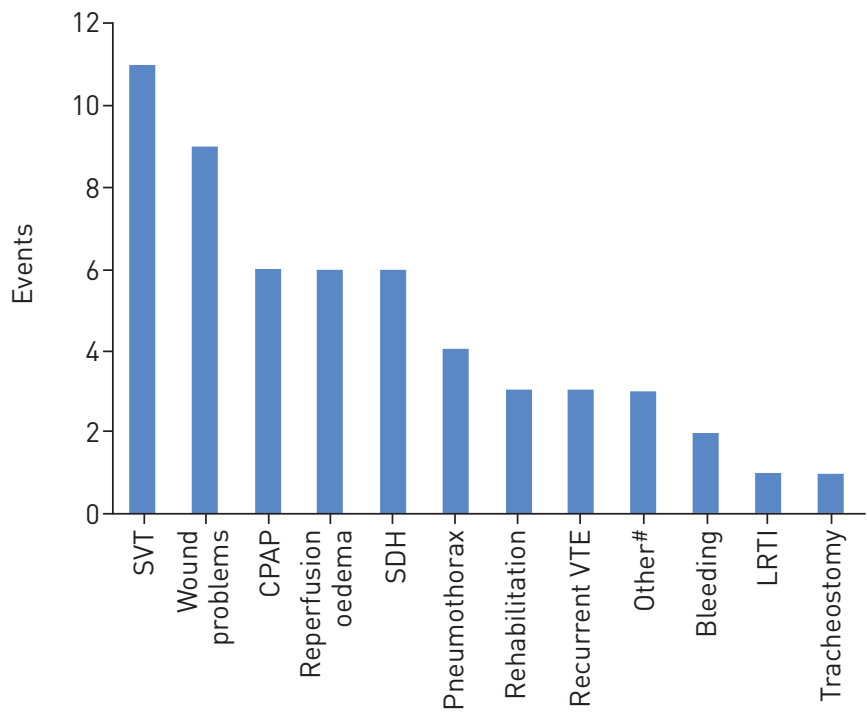

post-PEA (91 (6)\% at baseline versus 93 (5)\% at 6-months post-PEA (nonsignificant) and 93 (3)\% at 1-year post-PEA $(\mathrm{p}=0.008))$.

\section{Discussion}

CTEPH is a frequently unrecognised complication of VTE affecting up to one in 25 patients within 2 years of a pulmonary embolism episode [17-20]. However, the number of patients who exhibit persistent perfusion defects on imaging tests at 11 months may be as high as 50\% [21]. Therefore, some of the latter may well have CTED and symptoms that are trivialised when no echocardiographic evidence of $\mathrm{PH}$ is found.

TABLE 5 Post-operative outcomes at 6-months and 1-year post pulmonary endarterectomy (PEA)

Baseline 6-months post-PEA

1-year post-PEA

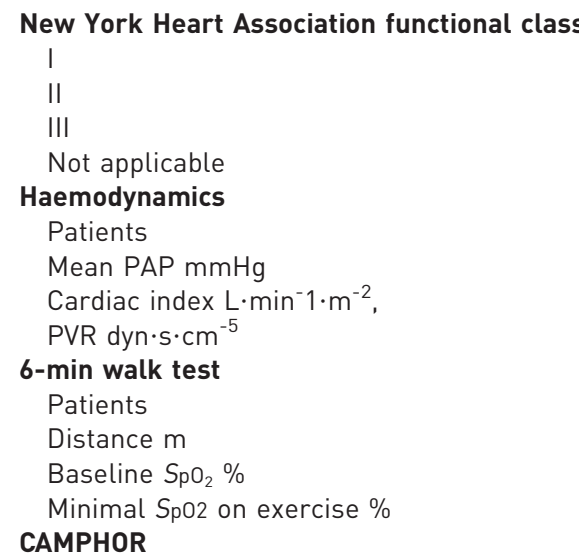

Patients

Total score

Symptoms

Activity

Quality of life

$\begin{array}{cc}0 & 16^{*} \\ 20 & 21^{*} \\ 22 & 2^{*} \\ 0 & 0 \\ 42 & 38^{\#} \\ 21(5) & 18(5)^{*} \\ 2.6 \pm 0.5 & 2.5 \pm 0.4 \\ 164(104) & 128(60)^{*}\end{array}$

$\begin{array}{ccc}37 & 34 & 27 \\ 372 \pm 117 & 413 \pm 90^{*} & 421 \pm 113^{*} \\ 97 \pm 2 & 97 \pm 2 & 97 \pm 2 \\ 91(6) & 93(5) & 93(3)^{*}\end{array}$

$\begin{array}{ccc}36 & 31 & 27 \\ 40(33) & 11(30)^{*} & 11(37)^{*} \\ 15(13) & 4(12)^{*} & 5(12)^{*} \\ 10(9) & 5(6)^{*} & 4(1)^{*} \\ 14(14) & 2(11)^{*} & 1(12)^{*}\end{array}$

Data are presented as $n$, median (interquartile range) or mean \pm SD. PAP: pulmonary arterial pressure; PVR: pulmonary vascular resistance; $\mathrm{SpO}_{2}$ : peripheral oxygen saturation; CAMPHOR: Cambridge pulmonary hypertension outcome review. ${ }^{\#}$ : one patient refused to have a repeat right heart catheterisation at the 6month post-surgical review. *: $\mathrm{p}<0.05$ compared to baseline. 
Quality of life

Total score

- Symptoms

Activity
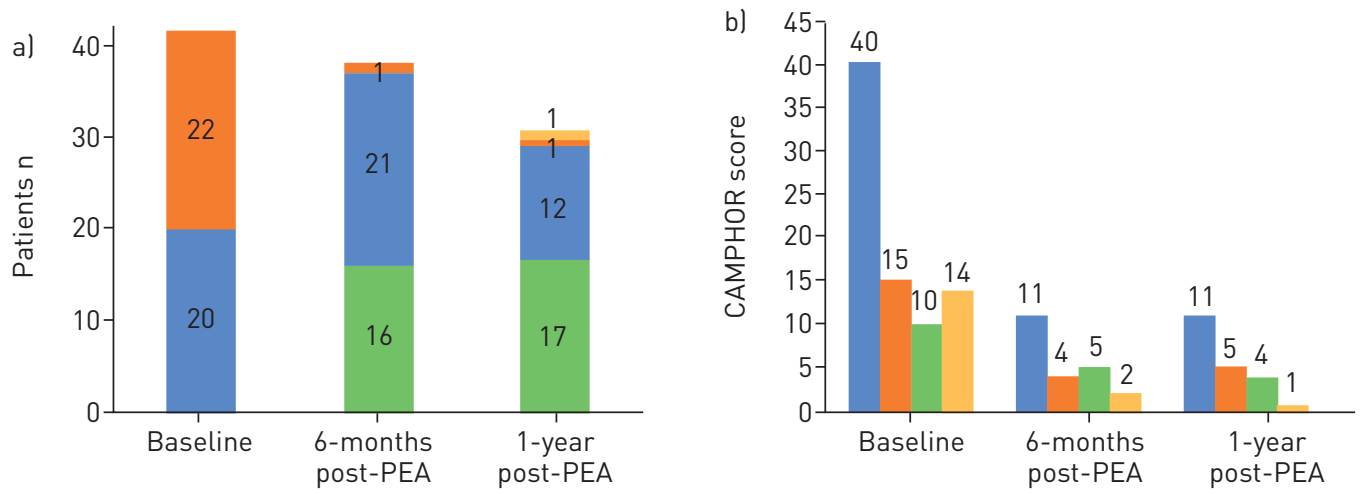

FIGURE 3 Individual changes in patient outcomes with respect to a) New York Heart Association (NYHA) functional class and b) Cambridge pulmonary hypertension outcome review (CAMPHOR) score during the study. PEA: pulmonary endarterectomy.

Our limited knowledge of the natural history of CTEPH is based on historical data prior to the current treatment era. Patients with mild PH (mean PAP $<30 \mathrm{mmHg}$ ) and "borderline PH" (mean PAP 21-24 mmHg) were thought to have a good prognosis, with low probability of progressing to severe $\mathrm{PH}$ when only treated with anticoagulation $[2,3]$, although these studies lack information on severity of the thrombotic burden. More recently a study on a small cohort of 10 patients with mild CTEPH reported improvement in functional status and good survival with conservative treatment. However, none of the patients had previous documented VTE and six were diagnosed with "inoperable" disease [22].

The present study focuses on CTED as a clinical entity where, despite extensive thromboembolic disease and mildly abnormal haemodynamics, no $\mathrm{PH}$ by the current threshold definition is found on RHC at rest. It has been established that $>60 \%$ of the pulmonary vasculature must be occluded to develop $\mathrm{PH}$ in acute thromboembolism [23], but severe $\mathrm{PH}$ out of proportion to vascular obliteration is a common finding in CTEPH and attributed to the development of secondary small vessel vasculopathy [24]. It is not clear why some patients develop PH and others do not, despite a similar burden of vascular occlusion seen on imaging. The incidence of CTED appears to be low in this series, representing $4 \%$ of the operable CTEPH patients.

Our cohort, as reported here, when compared to the CTEPH registry patients [6] is younger (49 years versus 60 years) and are more likely to have a previous pulmonary embolism (90\% compared to 79\%). One may speculate that our patients were diagnosed earlier in the course of the disease, thus they did not have time to develop PH. Nonetheless, this is not supported by the fact that the median time from last VTE to diagnosis was 14 months, similar to registry patients. We recognise that CTEPH is a heterogeneous disease. Demographic differences in our cohort may be explained by younger patients seeking medical attention when they fail to return to their fitness status before pulmonary embolism.
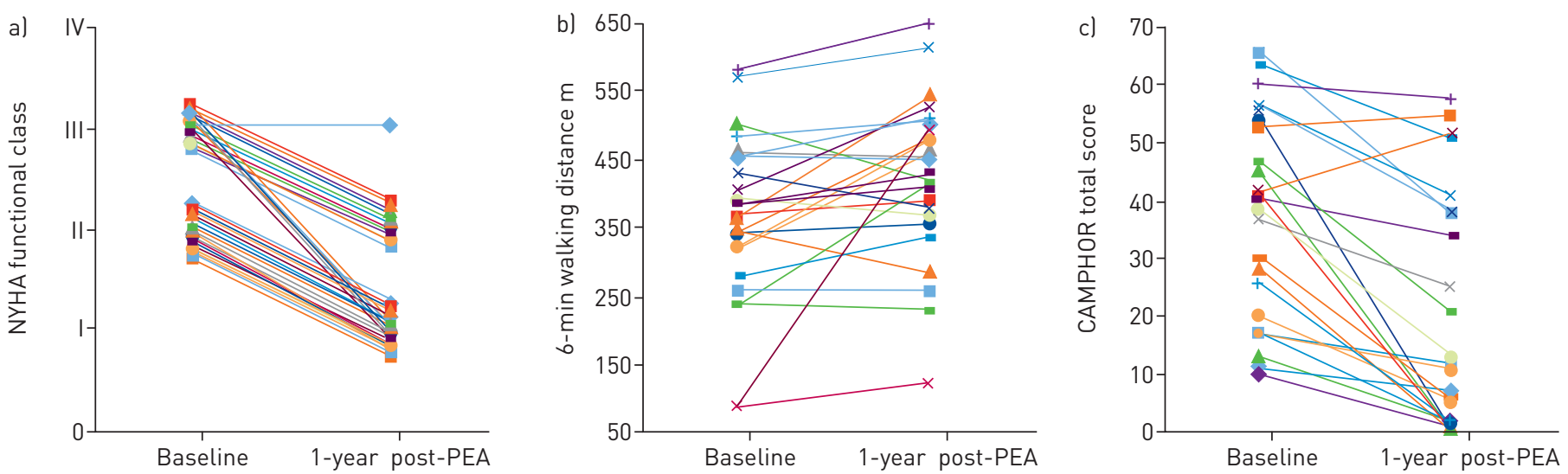

FIGURE 4 Individual changes in patient outcomes with respect to a) New York Heart Association (NYHA) functional class, b) 6-min walking distance, and c) Cambridge pulmonary hypertension outcome review (CAMPHOR) total score during the study. PEA: pulmonary endarterectomy. 
Our CTED patients reported exertional symptoms that seem out of proportion to their haemodynamics and right ventricular function. The majority of investigations were performed at rest, perhaps lacking the sensitivity to detect early small vessel vasculopathy or subclinical right ventricular impairment, though cardiac output was at the lower limit of normal even when at rest and patients significantly desaturated during the 6MWT (a submaximal exercise test). Increased physiological death space $(V \mathrm{D} / V \mathrm{~T})$ measured by cardiopulmonary exercise test (CPET) is known to be a major contributor to ventilatory inefficiency in CTEPH patients. This was described as a likely consequence of vascular occlusions and considered to be independent from haemodynamic impairment [25]. A small and selected group of patients from our cohort participated in a study looking at CPET in patients with thromboembolic disease. This study demonstrated that CTED patients have impaired right ventricular adaptation to exercise and inefficient gas exchange on exertion [26]. Moreover, our study on right ventricular mechanics using conductance catheter derived pressure-volume loops showed delayed right ventricular relaxation in these patients [27].

Pulmonary endarterectomy was offered to CTED patients with the aim to improve symptoms, exercise capacity and QoL with the hope of preventing disease progression given the extensive thrombotic burden. We have shown that surgical treatment can be safely performed in this group of patients without in-hospital mortality and 95\% 1-year survival rate. However, PEA is not a minor procedure and has considerable perioperative risk even when performed at an expert institution. Our rate of complications was consistent with other series $[6,9]$ and might be expected given the complexity of the surgical procedure and the patient's comorbidities.

Midterm outcome post-PEA was very good with significant improvement in symptoms, functional capacity, and QoL. Patient-reported outcome measures are increasingly being used as a measurement tool to assess treatment outcomes from the patient's perspective. We have observed a reduction in the median (IQR) score of 29 (3) points in the total CAMPHOR score with 11 (1), 5 (3) and 12(3) reduction in the symptoms, activity and QoL domains, respectively, reflecting a significant improvement in the patient's symptoms and QoL. This is comparable with the changes observed in CTEPH patients at 12 weeks and 52 weeks post-PEA reported in the PEACOG trial [14]. The total CAMPHOR score at diagnosis has previously been shown to be an independent predictor of clinical deterioration in CTEPH patients, with an increase in 10 points giving 51\% increase in the incidence of clinical deterioration [28].

With regards to exercise capacity, paired data analysis showed a $30 \mathrm{~m}$ (95\% CI 3-57 m) increase in mean walking distance at 6-months post-PEA and $54 \mathrm{~m}$ increase (95\% CI 16-93 m) at 1-year post PEA. The minimal important difference of the 6MWT has not been defined in CTEPH nor CTED patients, but is estimated at $33 \mathrm{~m}$ for PAH patients [29]. We know from the EARLY study (treatment of patients with mildly symptomatic pulmonary arterial hypertension with bosentan) that PAH patients who present at an earlier NYHA functional class show only a small improvement in the 6MWD with treatment [30].

The main limitation in our study is the lack of a comparative cohort of patients with CTED treated conservatively and long-term survival data comparing both patients whom had been operated on and patients that had not.

Early referral of CTED patients to an expert PEA centre for comprehensive assessment by a multidisciplinary CTEPH team is recommended. Objective measurements for exercise limitations using exercise catheterisation and a cardiopulmonary exercise test may help to confirm that symptoms are due to thromboembolic disease rather than comorbidities. Given that approximately one in 20 CTED patients treated with PEA may die within a year of surgery and presuming that historical patients with "borderline $\mathrm{PH}^{\prime}$ " are comparable to our cohort, conservative management is a safe choice for these patients having shown haemodynamic stability and a 95\% 5-year survival [2]. However, patients may not be prepared to live with persistent functional limitation and reduced QoL, and hence seek other treatment alternatives. Targeted medical therapy currently recommended for patients with inoperable CTEPH [31] has not established role in CTED patients with operable disease and a low probability of having significant small vessel vasculopathy. Emergent interventional techniques such as percutaneous balloon angioplasty [32-35] might have a future role in the treatment of CTED patients, but more robust data on safety and long-term outcomes is needed.

In summary, we conclude that in this carefully selected and individually counselled cohort of CTED patients with extensive operable thromboembolic disease, PEA resulted in symptomatic improvement, better functional status and QoL with associated morbidity and mortality similar to CTEPH patients. Given the perioperative complications and the fact that their prognosis without treatment is probably favourable, surgical treatment of CTED should only be performed in expert centres. Further research is required on the natural history of CTED and detailed discussion of the current evidence including risk benefit ratio of each treatment option on an individual patient basis is recommended. 


\section{Acknowledgements}

The authors would like to acknowledge our pulmonary endarterectomy specialist nurses, Anie Ponnaberanam and Maureen Rootes (Papworth Hospital, Cambridge, UK) for help with prospective data collection. We are indebted to the contributions to patient care made by the referring pulmonary hypertension physicians in the UK and Ireland.

\section{References}

1 Moser KM, Auger WR, Fedullo PF. Chronic major-vessel thromboembolic pulmonary hypertension. Circulation 1990; 81: 1735-1743.

2 Riedel M, Stanek V, Widimsky J, et al. Longterm follow-up of patients with pulmonary thromboembolism. Late prognosis and evolution of hemodynamic and respiratory data. Chest 1982; 81: 151-158.

3 Lewczuk J, Piszko P, Jagas J, et al. Prognostic factors in medically treated patients with chronic pulmonary embolism. Chest 2001; 119: 818-823.

4 Galiè N, Hoeper MM, Humbert M, et al. Guidelines for the diagnosis and treatment of pulmonary hypertension. Eur Heart J 2009; 30: 2493-537.

5 Jenkins D, Madani M, Mayer E, et al. Surgical treatment of chronic thromboembolic pulmonary hypertension. Eur Respir J 2013; 41: 735-742.

6 Mayer E, Jenkins D, Lindner J, et al. Surgical management and outcome of patients with chronic thromboembolic pulmonary hypertension: results from an international prospective registry. J Thorac Cardiovasc Surg 2011; 141: 702-710.

$7 \quad$ Health and Social Care Information Centre. National Pulmonary Hypertension Audit-2013. http://www.hscic.gov. uk/catalogue/PUB13318 Date last accessed: March 10, 2014. Date last updated: January 10, 2014.

8 Freed D, Thompson B, Berman M, et al. Survival after pulmonary thromboendarterectomy: effect of residual pulmonary hypertension. J Thorac Cardiovasc Surg 2011; 141: 383-387.

9 Berman M, Hardman G, Sharples L, et al. Pulmonary endarterectomy: outcomes in patients aged $>70$. Eur J Cardiothorac Surg, 41: e154-e160.

10 Madani MM, Auger WR, Pretorius V, et al. Pulmonary endarterectomy: recent changes in a single institution's experience of more than 2,700 patients. Ann Thorac Surg 2012; 94: 97-103.

11 Klepetko W, Mayer E, Sandoval J, et al. Interventional and surgical modalities of treatment for pulmonary arterial hypertension. J Am Coll Cardiol 2004; 43: Suppl. S73-S80.

12 Mayer E. Surgical and post-operative treatment of chronic thromboembolic pulmonary hypertension. Eur Respir Rev 2010; 19: 64-67.

13 Fedullo P, Kerr KM, Kim NH, et al. Chronic thromboembolic pulmonary hypertension. Am J Respir Crit Care Med 2011; 183: 1605-1613.

14 Vuylsteke A, Sharples L, Charman G, et al. Circulatory arrest versus cerebral perfusion during pulmonary endarterectomy surgery (PEACOG): a randomised controlled trial. Lancet 2011; 378: 1379-1387.

15 Jamieson SW, Kapelanski DP, Sakakibara N, et al. Pulmonary endarterectomy: experience and lessons learned in 1,500 cases. Ann Thorac Surg 2003; 76: 1457-1462.

16 McKenna SP, Doughty N, Meads DM, et al. The Cambridge Pulmonary Hypertension Outcome Review (CAMPHOR): a measure of health-related quality of life and quality of life for patients with pulmonary hypertension. Qual Life Res 2006; 15: 103-115.

17 Pengo V, Lensing AW, Prins $\mathrm{MH}$, et al. Incidence of chronic thromboembolic pulmonary hypertension after pulmonary embolism. N Engl J Med 2004; 350: 2257-2264.

18 Becattini C, Agnelli G, Pesavento R, et al. Incidence of chronic thromboembolic pulmonary hypertension after a first episode of pulmonary embolism. Chest 2006; 130: 172-175.

19 Miniati M, Monti S, Bottai M, et al. Survival and restoration of pulmonary perfusion in a long-term follow-up of patients after acute pulmonary embolism. Medicine (Baltimore) 2006; 85: 253-262.

20 Klok FA, van Kralingen KW, van Dijk AP, et al. Prospective cardiopulmonary screening program to detect chronic thromboembolic pulmonary hypertension in patients after acute pulmonary embolism. Haematologica 2010; 95: 970-975.

21 Nijkeuter M, Hovens MM, Davidson BL, et al. Resolution of thromboemboli in patients with acute pulmonary embolism: as systemic review. Chest 2006; 129: 192-197.

22 Romaszkiewicz R, Lewczuk J, Piszko P, et al. Clinical course of unoperated mild chronic thromboembolic pulmonary hypertension. Kardiol Pol 2011; 69, 5: 438-443.

23 Azarian R, Wartski M, Collignon MA, et al. Lung perfusions scans and hemodynamics in acute and chronic pulmonary embolism. J Nucl Med 1997; 38: 980-983.

24 Moser KM, Blood CM. Pulmonary vascular lesions occurring in patients with chronic major vessel thromboembolic pulmonary hypertension. Chest 1993; 103: 685-692.

25 Zhai Z, Murphy K, Tighe H, et al. Differences in ventilatory inefficiency between pulmonary arterial hypertension and chronic thromboembolic pulmonary hypertension. Chest 2011; 140: 1284-1291.

26 McCabe C, Deboeck G, Harvey I, et al. Inefficient exercise gas exchange identifies pulmonary hypertension in chronic thromboembolic obstruction following pulmonary embolism. Thromb Res 2013; 132: 659-665.

27 McCabe C, White PA, Hoole SP, et al. Right ventricular dysfunction in chronic thromboembolic obstruction of the pulmonary artery. J Appl Physiol (1985) 2014; 116: 355-363.

28 McCabe C, Bennett M, Doughty N, et al. Patient- reported outcomes assessed by the CAMPHOR questionnaire predicts clinical deterioration in idiopathic pulmonary arterial hypertension and chronic thromboembolic pulmonary hypertension. Chest 2013; 144: 522-530.

29 Mathai SC, Puhan MA, Lam D, et al. The minimal important difference in the 6-minute walk test for patients with pulmonary arterial hypertension. Am J Respir Crit Care Med 2012; 186: 428-433.

30 Galiè N, Rubin LJ, Hoeper M, et al. Treatment of patients with mildly symptomatic pulmonary arterial hypertension with bosentan (EARLY study): a double-blind, randomised controlled trial. Lancet 2008; 371: 2093-2100.

31 Kim NH, Delcroix M, Jenkins DP, et al. Chronic thromboembolic pulmonary hypertension. J Am Coll Cardiol 2013; 62: Suppl. D92-D99. 
32 Feinstein JA, Goldhaber SZ, Lock JE, et al. Balloon pulmonary angioplasty for treatment of chronic thromboembolic pulmonary hypertension. Circulation 2001; 103: 10-13.

33 Sugimura K, Fukumoto Y, Satoh K, et al. Percutaneous transluminal pulmonary angioplasty markedly improves pulmonary hemodynamics and long-term prognosis in patients with chronic thromboembolic pulmonary hypertension. Circ J 2012; 76: 485-488.

34 Kataoka M, Inami T, Hayashida K, et al. Percutaneous transluminal pulmonary angioplasty for the treatment of chronic thromboembolic pulmonary hypertension. Circ Cardiovasc Interv 2012; 5: 756-762.

35 Mizoguchi H, Ogawa A, Munemasa M, et al. Refined balloon pulmonary angioplasty for inoperable patients with chronic thromboembolic pulmonary hypertension. Circ Cardiovasc Interv 2012; 5: 748-755. 